\title{
A NEW LEAKY-WAVE DIRECTIONAL COUPLER IN HYBRID DIELECTRIC-WAVEGUIDE PRINTED-CIRCUIT TECHNOLOGY
}

\author{
José Luis Gómez-Tornero, Sofía Martínez, David Cañete, Juan Pascual and Alejandro Álvarez-Melcón \\ Department of Information and Communications Technologies, Technical University of Cartagena \\ 30202 Cartagena, Spain
}

\begin{abstract}
This paper presents the design of a novel leakywave coupler, based on hybrid dielectric-waveguide printedcircuit technology. The working mechanism of the proposed structure is explained, and the design procedure is illustrated in order to obtain the geometrical dimensions for a coupler working at 5.5GHz. The design is performed using commercial electromagnetic analysis software. This novel directional coupler presents interesting advantages when compared to previous wave couplers.
\end{abstract}

Index Terms - Directional Coupler, Leaky-Waves.

\section{INTRODUCTION}

A directional coupler is an essential subsystem for most communications systems in both optic and microwave ranges [1]. Figure 1 illustrates different coupling mechanisms between two dielectric guides. In Fig.1-a, the proximity coupling mechanism is sketched, showing how the evanescent fields outside the dielectric slab can couple from the input to the coupled guide, provided the distance $L$ is short enough [2]. In some applications, a coupling higher than $-6 d B$ is desired, requiring the gap $L$ to be too small to be implemented in practical circuits. This problem is even more serious as frequency is increased, due to the small dimensions and critical tolerances involved in the fabrication processes.

A coupling mechanism which overcomes the problem associated to the gap distance $L$ is the radiation coupling mechanism [3]. The initially bounded mode of the dielectric guide is transformed into a leaky-mode by inserting some kind of discontinuities. The energy is radiated from the input guide to the coupled guide at a given angle, as shown in Figs.1-b and 1-c. Different topologies have been conceived to induce and control this leaky-mode radiation in the microwave range, using dielectric strips [3],[4] and NRD guide technology [5]. In the optic range, several studies have been performed to conceive optic couplers using dielectric guides and prisms [6]. The most well-known leaky-wave coupler in the optic range is the so called "grating coupler" [7-8] shown in Fig.1-b. The periodic discontinuities inserted in the dielectric guide excite the $m=-1$ radiating space-harmonic. This harmonic is used as a radiating backward leaky-wave, which couples the energy from the input port to the coupled port, as shown in Fig.1-b. The grating must be designed so that all the energy radiated is absorbed by the receiving grating, minimizing the scattered energy (maximum coupling efficiency) [7,8].
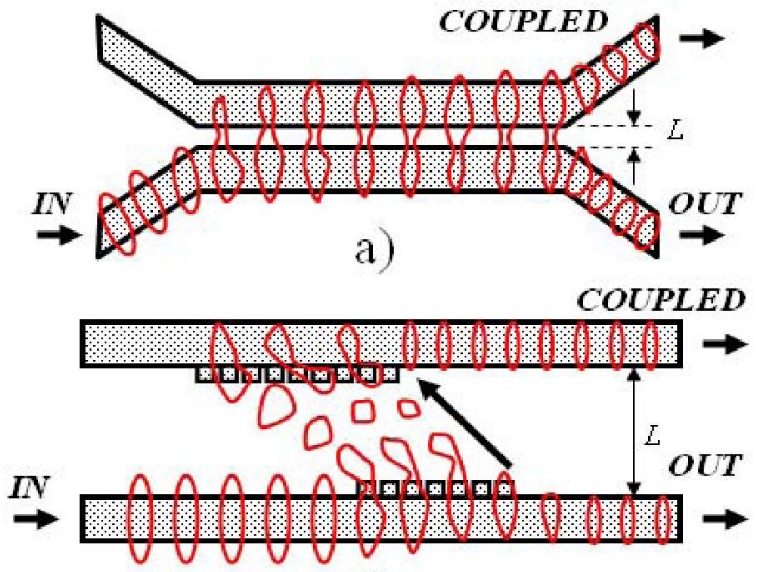

b)

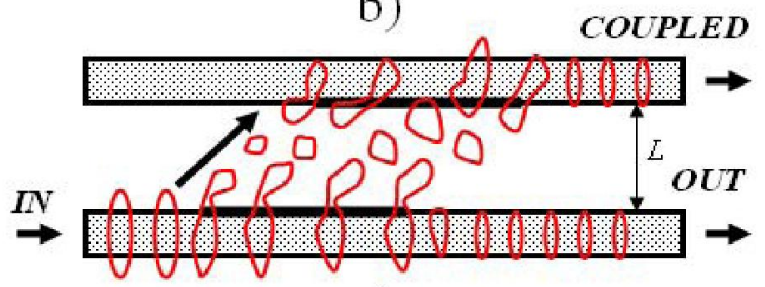

c)

Fig.1. Different types of directional couplers

In this paper, a new leaky-wave coupler is presented, together with the design procedure to obtain coupling under maximum efficiency conditions. There are two main advantages with respect to previous leaky-wave couplers. First, the new coupler is based on the radiation of the main harmonic (forward leaky-wave) as it can be seen in Fig.1-c. In this way the dimensions of the structure are minimized, since it is not necessary to excite higher-order space-harmonics. This point is of much importance in the microwave frequency range, where the dimensions of these couplers can be quite large. The second advantage is related to the fact that the radiation is induced and controlled by the use of a slot-circuit, which is printed on the broad side of the dielectric guide. This hybrid printed-circuit dielectric-guide technology [9] is much easier to manufacture and more flexible to design than the conventional grating-coupler. The complicated mechanization of the dielectric waveguide is avoided, while the printed circuits can be manufactured using standard photolithographic processes. 


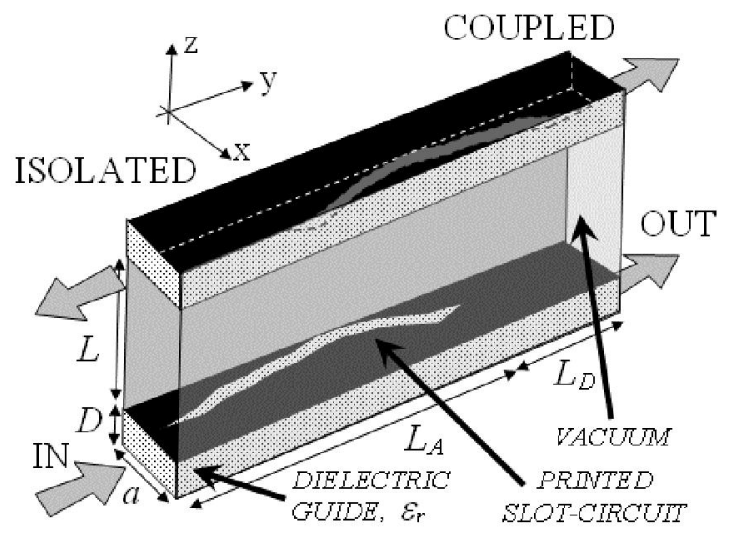

Fig.2. Scheme of the Proposed Leaky-Wave Coupler

\section{DESIGN PROCEDURE}

In this section, the working mechanism of the proposed coupler will be explained, illustrating the mission of each part of the circuit and the design procedure which must be followed to obtain a successful response. The scheme of the coupler, including the main dimensions, is shown in Fig.2.

\section{A. Dielectric Guides and Separation Height L}

Rectangular dielectric waveguides of width $a$, height $D$ and relative permittivity $\varepsilon_{r}$ are used to propagate the main $\mathrm{TE}_{10}$ mode. These dimensions control the cutoff frequency of the $\mathrm{TE}_{10}$ mode, and therefore the fast-wave frequency region where this mode can become leaky. This region also depends on the printed-circuit added at the dielectric-air interface, as it can be seen in Fig.3. In the figure is plotted the normalized phase constant $\left(\beta / k_{0}\right)$ of the $\mathrm{TE}_{10}$ mode, for three different slot widths $W$ (see inset in Fig.3).
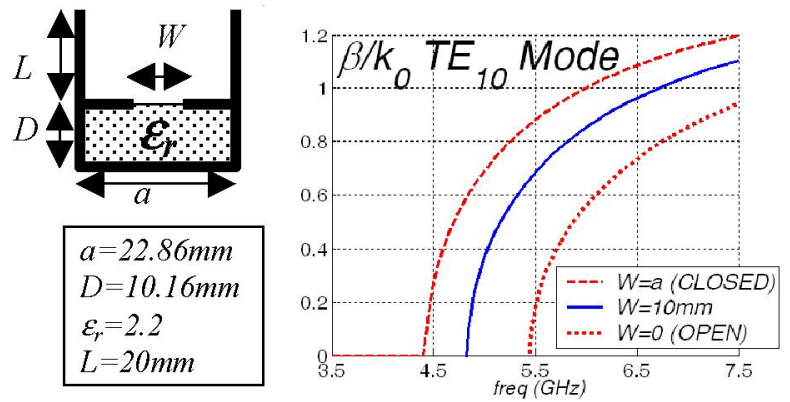

Fig.3. Normalized phase constant of $\mathrm{TE}_{10}$ mode

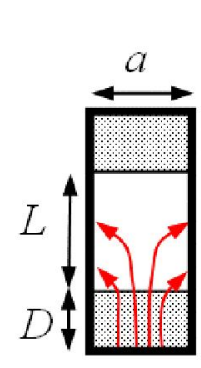

a)

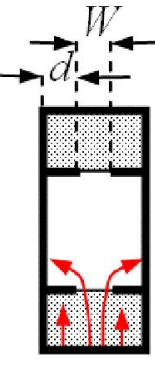

b)

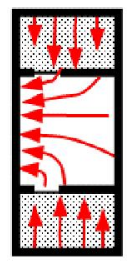

c)
Fig.4 Asymmetry radiation and coupling mechanism
As it can be seen in Fig.3, the fast-wave region of the $\mathrm{TE}_{10}$ mode extends from $4.8 \mathrm{GHz}$ to $6.5 \mathrm{GHz}$ for a dielectric guide of dimensions $a=22.86 \mathrm{~mm}, D=10.16 \mathrm{~mm}, \varepsilon_{r}=2.2$, and a centered slot of width $W=10 \mathrm{~mm}$. In addition, the slot must be uncentered with respect to the parallel plates (see Fig.2), in order to launch the leaky-wave. This asymmetry radiation mechanism is explained in detail in [9], where interesting leaky-wave antennas (LWAs) were designed using this technology. In this way, no coupling will occur between the two dielectric guides if the separation $L$ between them is large enough, and the printed slot-circuit is centered, as sketched in Figs.4-a and 4-b. However, if the slot is uncentered, there will be radiation and coupling no matter the value of $L$, as illustrated by Fig.4-c.

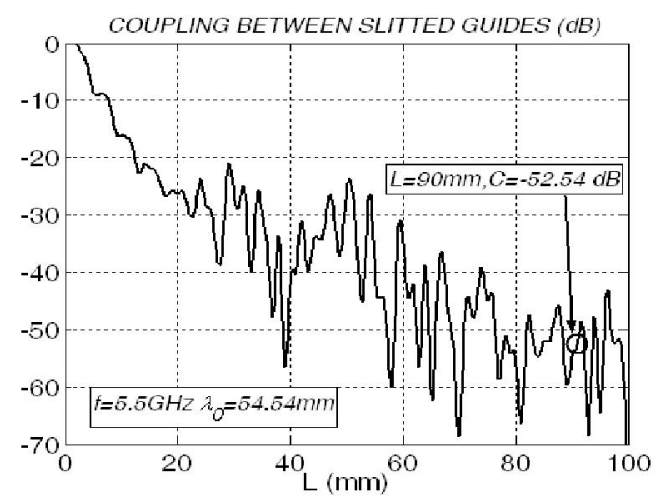

Fig.5. Election of the separation $L$ to avoid direct coupling

Figure 5 shows the results obtained from HFSS for the coupling between two guides with a centered slot of width $W=10 \mathrm{~mm}$, as a function of the separation $L$. It can be seen that with $L=90 \mathrm{~mm}$ the direct coupling due to the evanescent surface-wave is very low $(-52 \mathrm{~dB})$. Consequently, coupling can now only be obtained by exciting a leaky-wave mode in the structure (by asymmetrically placing the slot).

\section{B. Printed Circuit Design}

The asymmetrical printed-circuit slot is responsible for inducing and controlling the leaky-wave radiation, and therefore plays a fundamental role in the design of the coupler. Particularly, the slot will determine the shape of the radiated beam. If the slot-circuit is uniform (see Fig.6-a), an exponential beam will be created. The coupling between the transmitter (TX) and the receiver (RX) guides will not be very efficient, since the overlapping integral [8] will not be maximum, as illustrated in Fig.6-a.
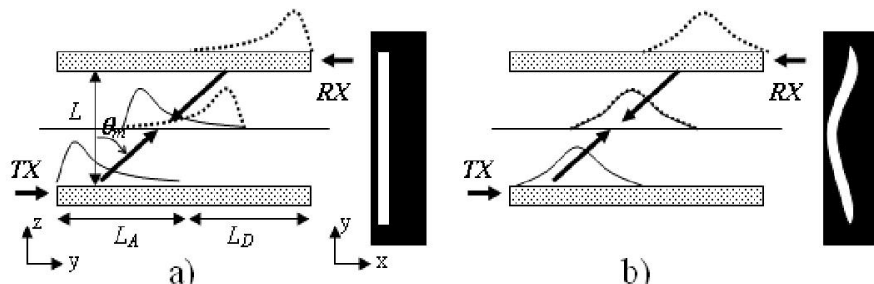

Fig.6 Necessity of tapering the printed circuit 
The printed-circuit must be tapered to obtain maximum coupling efficiency [8]. Using $\mathrm{TE}_{10}$ leaky-mode dispersion curves [9], the dimensions of the slot circuits are designed to synthesize cosine-shaped linear-phase radiated beams of length $L_{A}=10 \lambda_{0}=545.45 \mathrm{~mm}$, as illustrated in Fig.6-b.

\section{Longitudinal Offset between Couplers, $L_{D}$.}

The cosine-shaped beams are radiated at a given angle $\theta_{m}$, which must be the same for all the antenna length. The angle $\theta_{m}$ depends on the leaky-mode normalized phase constant:

$$
\sin \theta_{m}=\frac{\beta}{k_{0}}
$$

For a given central frequency, which in our case is $5.5 \mathrm{GHz}$, the TX and RX slot circuits of length $L_{A}$ must be displaced an offset $L_{D}$ (see Fig.2 and Fig.6-a) in order to make the emitted beam overlap with the trace of the receiver [8]. The theoretical value of $L_{D}$ which provides this overlapping is given by:

$$
L_{D}=L \cdot \tan \theta_{m}
$$

In our case, $L=90 \mathrm{~mm}$ and the radiating angle obtained from the $\mathrm{TE}_{10}$ leaky-mode dispersion curve is $\theta_{m}=40^{\circ}$, which gives raise to an offset $L_{D}=75.51 \mathrm{~mm}$. Figure 7 -a shows the variation of the coupling as a function of the longitudinal offset between couplers $L_{D}$ for the designed cosine-tapered slotcircuits at $5.5 \mathrm{GHz}$, obtained with HFSS. It is checked that the maximum coupling takes place between $70 \mathrm{~mm}$ and $80 \mathrm{~mm}$.
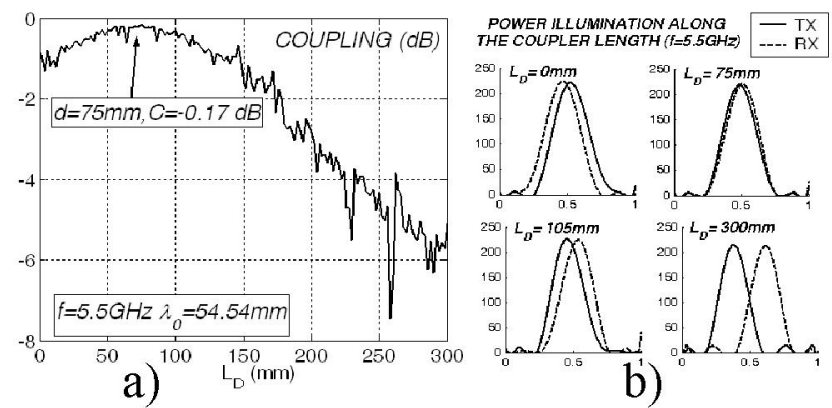

Fig.7 Variation of Coupling with TX-RX Longitudinal Offset $L_{D}$

In Fig.7-b, the cosine beams emitted by the TX and the RX LWAs along the normalized coupler length are obtained at the middle of the coupler height ( $\mathrm{z}=L / 2$, see Fig.2), confirming that the maximum overlapping is produced for $L_{D}=75 \mathrm{~mm}$. For other values of $L_{D}$ the traces are shifted, obtaining a smaller overlapping integral, which is translated into a lower coupling [8], as it can be seen in Fig.7-a.

\section{Frequency Response}

When frequency is varied around the design point $(5,5 \mathrm{GHz})$, a similar beam shifting phenomenon occurs. Due to the frequency scanning response of LWAs, the beams are displaced and the overlapping is reduced, as show in Fig.8. Fig.9 shows the coupling obtained with HFSS for the designed directional coupler in the range from $4.5 \mathrm{GHz}$ to $8 \mathrm{GHz}$.

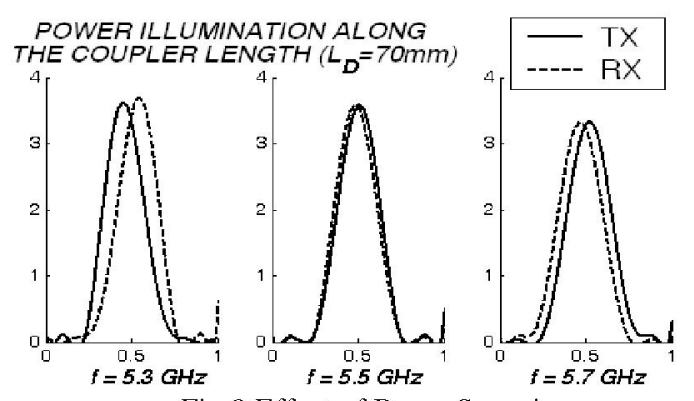

Fig.8 Effect of Beam-Scanning

Three different operating regions can be observed in Fig.9. Below $4.8 \mathrm{GHz}$, the leaky- $\mathrm{TE}_{10}$-mode is below cutoff, as it was shown in Fig.2 for the slitted waveguide with $W=10 \mathrm{~mm}$. Also it was shown in Fig.2 that the leaky-wave region corresponds to the range between $4.8 \mathrm{GHz}$ and $6.5 \mathrm{GHz}$, In Fig. 9 it is verified that the coupling occurs in this region. Above $6.5 \mathrm{GHz}$ the $\mathrm{TE}_{10}$ mode becomes a surface-wave, making most of the injected energy to travel to the OUT port (see Fig.2). The return losses and the isolation are below $-15 \mathrm{~dB}$ in the entire coupling band, and are not shown here due to space limitation.

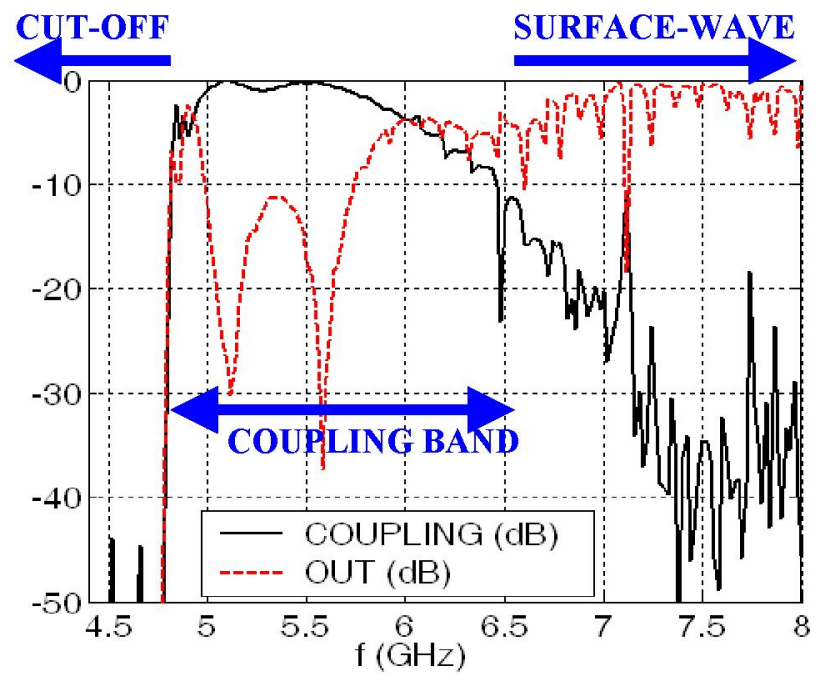

Fig.9 Frequency Response of Designed Coupler obtained with HFSS

In the coupling band, it is observed that the maximum coupling is obtained at the design frequency $(5.5 \mathrm{GHz})$. Around this frequency, the coupling decreases due to the frequency beam shifting phenomenon illustrated in Fig, 8 . However, another maximum appears at $5.1 \mathrm{GHz}$. This maximum is created by a second order overlapping, illustrated in Fig.10-a. The TX and RX beams overlap after some reflections, which are only produced at low frequencies due to the nearly-normal angle of emission $\theta_{m}$. For frequencies above $5.5 \mathrm{GHz}$, the emitted beams diverge (see Fig.10-b) and coupling decreases as frequency increases, as it can be seen in Fig.9. Absorbing materials at the ends of the coupler must be used to avoid interferences, which would be created by the reflection of these waves in the upper frequency region. 


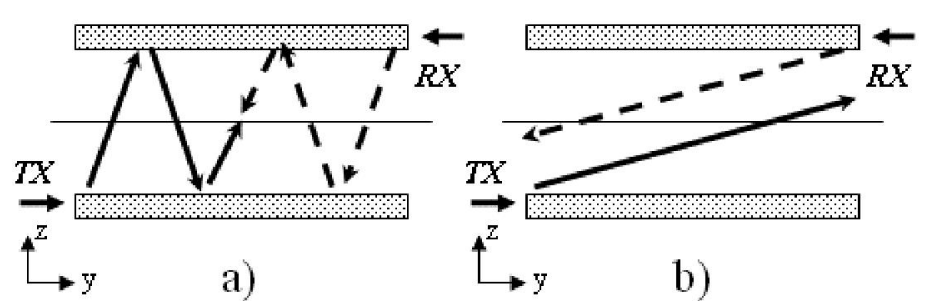

Fig.10 a) Higher-order Coupling Phenomenon at Low Frequencies b) Divergence Phenomenon at High Frequencies

\section{E. Control of Coupling Level}

All the previous results were obtained for a coupler designed to obtain a coupling level of $C=-0.2 d B$ at the design frequency of $5.5 \mathrm{GHz}$. The maximum coupling level can be controlled by the radiation efficiency of the TX and RX LWAs, namely $\eta^{T X}$ and $\eta^{R X}$. For the frequency in which the overlapping integral is maximized (maximum coupling efficiency, in our case at $5.5 \mathrm{GHz}$ ), the coupling level can be computed as:

$$
C=\eta^{T X} \cdot \eta^{R X}
$$

The LWAs radiation efficiency $\eta$ can be computed from the variation of the leaky-mode leakage rate along the radiating antenna length $L_{A}, \alpha(y)$ :

$$
\eta=1-e^{-2 \cdot \int_{y=0}^{y=L_{A}} \alpha(y) \cdot d y}
$$

If the RX LWA is designed with maximum radiation efficiency $\left(\eta^{R X} \approx 1\right.$ ) the coupling level can be determined by the TX LWA efficiency, $\eta^{T X}$. In this way, the input guide printedcircuit will be responsible for controlling the coupling level of the directional coupler. Figure 11 shows the results obtained with HFSS for three couplers with maximum overlapping designed at $5.5 \mathrm{GHz}$. One coupler is designed for a $95 \%$ coupling level $\left(\eta^{T X}=\eta^{R X}=0.97, C=0.95=-0.2 d B\right)$, other for $52 \%$ of coupling $\left(\eta^{R X}=0.97, \eta^{T X}=0.53, C=0.52=-2.8 d B\right)$ and the last one for a coupling level of $52 \% \quad\left(\eta^{R X}=0.97, \quad \eta^{T X}=0.23\right.$ $C=0.22=-6.6 d B)$.

\section{Conclusion}

A new leaky-wave directional coupler in hybrid dielectricwaveguide printed-circuit technology has been presented. The dielectric guide dimensions control the fast-wave frequency band of the $\mathrm{TE}_{10}$ mode. The slot printed-circuit controls the leakage rate, therefore being responsible for synthesizing a cosine shaped beam to obtain maximum coupling efficiency (maximum overlapping integral). The offset between the transmitter and the receiver leaky-wave radiating slots must be chosen to maximize the overlapping at the design frequency. Finally, the printed-circuit also controls the radiation efficiency of the transmitter slot, allowing for the adjustment of the coupling level. The proposed coupler presents a more flexible and simpler design than conventional grating couplers, since the dielectric guide structure must not be mechanized to obtain a maximum efficiency design. Only the printed-circuit must be tapered. In addition, the new coupler is based on radiation from the main space-harmonic. In this way the structure dimensions are minimized if compared with grating couplers, which are based on higher-order radiating harmonics.

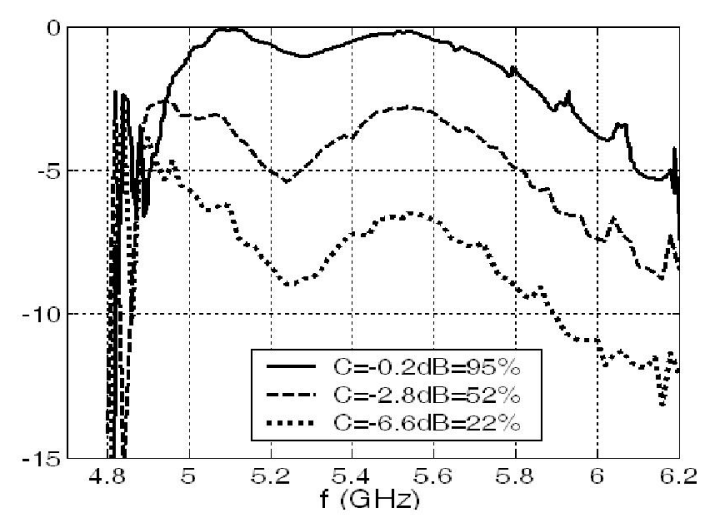

Fig.11 Frequency Response for Different Coupling Levels

\section{ACKNOWLEDGEMENT}

This work has been partially supported by Spanish national projects ESP2001-4546-PE, TEC2004-04313-C02-02 and regional Séneca project 2002 PB/4/FS/02.

\section{REFERENCES}

[1] I. Bahl and P. Bhartia, Microwave Solid State Circuit Design, Ed. John Wiley \& Sons, $2^{\text {nd }}$ Ed., 2003, Ch.5, pp.209-240.

[2] T. Yoneyama, N. Tozawa and S. Nishida, "Coupling Characteristics of Nonradiative Dielectric Waveguides", IEEE Trans. Microwave Theory Techn., Vol.31, pp.648-654, August 1983.

[3] T. Yoneyama and S. Nishida, "Dielectric Waveguide Coupling by Leaky Wave", in Proc. Int. Symp. Microwave and Commun., India, pp. 185-190, December 1981.

[4] E. W. Hue, S. T. Peng and A. A. Oliner, "A Novel Leaky-Wave Strip Waveguide Directional Coupler", in Topical Meet. Integrated and Guided Wave Opt., Salt Lake City, UT, paper WD2, January 1978.

[5] D.-C. Niu, T. Yoneyama and T. Itoh, "Analysis and Measurement of NRD-Guide Leaky Wave Coupler in Ka Band", IEEE Trans. Microwave Theory Techn., Vol.41, No.12, pp.2126-2132, Dec. 1993.

[6] T. Tamir, Integrated Optics, Topics in Applied Physics 7, Springer Verlag, 1975, Chapter 3, p.84.

[7] T. Tamir and S. T. Peng, "Analysis and design of grating couplers", Appl. Phys., Vo. 14, pp.235-254, 1977.

[8] R. Ulrich, "Efficiency of optical-grating couplers", J. Opt. Soc. Am. , Vol.63, No.11, pp. 1419-1431, November 1973.

[9] J.L. Gómez, A. de la Torre, D. Cañete, M. Gugliemi and A.A. Melcón, "Design of Tapered Leaky-Wave Antennas in Hybrid Waveguide-Planar Technology for Millimeter Waveband Applications", IEEE Trans. Antennas Propagat., Vol.53, No.8, pp.2563-2577, August 2005. 